\title{
Post-Test Probability for Pulmonary Embolism after Ventilation-Perfusion Scan Assessed by Nuclear Medicine Physicians
}

\author{
Abdelhamid H. Elgazzar ${ }^{\mathrm{b}}$ Issa Loutfi ${ }^{\mathrm{b}}$ Mona Baqer ${ }^{\mathrm{c}}$ M. Farghali ${ }^{\mathrm{c}}$ \\ Mousa Khadada ${ }^{a}$ Sherif Heiba $^{b}$ Omash Uza ${ }^{d}$ Suad Mahussein ${ }^{c}$ \\ Samira Al-Tailjic \\ Departments of ${ }^{a}$ Medicine and ${ }^{b}$ Nuclear Medicine, Faculty of Medicine, Kuwait University, ${ }^{\mathrm{c}}$ Mubarak Al-Kabeer \\ Hospital, Ministry of Health, Safat, Kuwait; ${ }^{\mathrm{d}}$ Tufts New England Medical Center, Boston, Mass., USA
}

\section{Key Words}

Pulmonary embolism • Pre- and post-scan probability • Ventilation-perfusion scan

\begin{abstract}
Objective: The objective of this prospective study was to compare assessments of pre- and post-ventilation-perfusion (V/Q) scan probabilities for pulmonary embolism (PE) by the nuclear medicine physician and the referring physician. Materials and Methods: Seventy-nine patients (41 females, 38 males, mean age 49 years) referred for V/Q scans over a period of 6 months and suspected of PE were included in the study. The pre-test likelihood was determined independently by a consultant nuclear physician and a referring physician prior to the $\mathrm{V} / \mathrm{Q}$ scan. The scan was performed according to a standard protocol using 8 view technetium-99m diethylenetriamine pentaacetic acid aerosol ventilation study followed by a technetium-99m macroaggregated albumin perfusion study. The scan was interpreted according to prospective investigation of pulmonary embolism diagnosis criteria. The post-scan probability was calculated. The probability of PE was quoted as low, intermediate and high. Agreement or disagreement in assessing the preand post-scan probability was classified according to the degree of difference (no difference: 0; minor difference:
\end{abstract}

1, and major difference: 2 grades). Results: The agreement between the nuclear medicine and referring physicians on clinical probability of PE was moderate $(63 \%)$ before the scan and good (90\%) after the scan. The disagreement in assessing the clinical probability between the referring physician and the nuclear medicine physician was predominantly minor. Only 5 and $1 \%$ of the disagreement was major in pre-scan and post-scan probabilities, respectively. Conclusion: The data show that nuclear medicine physicians can use the assessment of pre-scan clinical likelihood to determine the post-scan probability of $\mathrm{PE}$.

Copyright $(2005$ S. Karger AG, Basel

\section{Introduction}

Scintigraphy is a suitable and cost-effective modality for the diagnosis of pulmonary embolism (PE). Due to the nonspecificity of the findings of the ventilation-perfusion (V/Q) scan, a probability for the likelihood of PE is reported. The scan probability of PE depends predominantly on the size and number of segmental perfusion defects and the corresponding ventilation pattern. The larger and more numerous the segmental defects, and the more they mismatch the ventilation pattern, the higher the chance of PE [1-5].

\section{KARGER}

Fax +4161306 1234 E-Mail karger@karger.ch www.karger.com
Abdelhamid H. Elgazzar, MD, FCAP

Department of Nuclear Medicine, Faculty of Medicine Kuwait University, PO Box 24923

Safat, 13110 (Kuwait)

Tel. +965 53195 92, Fax +965 53389 36, E-Mail aelgazzar49@hotmail.com 
Usually, the scan probability for PE is reported by nuclear medicine physicians based on these scintigraphic patterns, without including the patient's clinical data, which represent the pre-scan probability or pre-scan clinical odds. This mode of assessment is consistent with the established observation that clinical data, except for history of prior cardiopulmonary disease, should not be used to determine the scan probability [6-7].

The decision-making process for managing patients suspected of having PE depends on the Bayes' theorem which relates the pre-scan clinical odds to scan findings to form the post-scan probability of PE [8-10]. Given a V/Q scan report, clinicians may misinterpret the findings in up to $35 \%$ of cases [11]. No objective criteria are available for the determination of the pre-scan probability by nuclear medicine physicians. Since this may help in the decision-making process in patient management, it is worth investigating whether the nuclear medicine physician can assess post-scan probability in addition to scan probability.

The aim of this prospective study was to determine whether or not nuclear physicians can assess and report the post-scan probability for PE, and whether or not this assessment is comparable to the evaluations of referring physicians (pre-scan probability).

\section{Subjects and Methods}

Seventy-nine patients ( 41 female and 38 male; mean age 49 years) referred for V/Q scan for suspicion of PE were included in the study. For all the cases, a ventilation study was performed first followed by a perfusion scan. The ventilation study was performed using 35-40 mCi (1,295-1,480 MBq) technetium-99m diethylenetriamine pentaacetic acid in a commercially available nebulizer system supplier. Imaging was conducted using a large field of view gamma camera equipped with a high resolution collimator. Images of 500 kilo counts (Kct) were obtained in posterior, left posterior oblique, left lateral, anterior, right lateral and right posterior oblique projections.

The perfusion images were performed after intravenous administration of $6 \mathrm{mCi}(22 \mathrm{MBq})$ of technetium- $99 \mathrm{~m}$ macro-aggregated albumin. Images were then acquired for 500-1,000 Kct in the same projections as the ventilation study.

A posterior-anterior chest radiograph obtained within $24 \mathrm{~h}$ of each study was available for review at the time of lung scan and the radiographic findings were incorporated in the interpretation of the scan.

The pre-scan probability was determined independently by both a nuclear physician and a referring physician. The physicians relied on the available clinical, radiologic and laboratory data obtained from the medical records of the patient. The data included age, sex, weight, symptoms and signs such as pain, hemoptysis, dyspnea and vital signs, history of DVT, postoperative status, can-
Table 1. Nuclear medicine and referring physicians' agrement on clinical probability of pulmonary embolism

\begin{tabular}{lllll}
\hline $\begin{array}{l}\text { Clinical } \\
\text { probability }\end{array}$ & Agreement & $\begin{array}{l}\text { Kappa } \\
\text { value }\end{array}$ & $\begin{array}{l}\text { Minor } \\
\text { disagreement }\end{array}$ & $\begin{array}{l}\text { Major } \\
\text { disagreement }\end{array}$ \\
\hline Pre-scan & $50 / 79$ & 0.423 & $25 / 79$ & $4 / 79$ \\
& $(63 \%)$ & $(0.081)$ & $(32 \%)$ & $(5 \%)$ \\
Post-scan & $71 / 79$ & 0.787 & $7 / 79$ & $1 / 79$ \\
& $(90 \%)$ & $(0.068)$ & $(9 \%)$ & $(1 \%)$ \\
\hline
\end{tabular}

Figures in parentheses in the kappa value column are standard errors.

cer, long term immobilization, cardiopulmonary disease, arterial blood gases and findings of echocardiac, ECG and current chest Xray. The pre-scan probability was classified according to the prospective investigation of pulmonary embolism diagnosis (PIOPED) criteria as follows: Low $=0-19 \%$; intermediate $=20-79 \%$, and high $=$ more than $80 \%$.

The scan probability of each case was then determined by a nuclear medicine physician, who was blinded to the clinical data, using the standard set of modified PIOPED criteria [4]. A consultant nuclear physician and a referring physician independently determined the post-scan probability using the pre-scan and scan probabilities. The same scale for reporting probability of PE was used for the pre-scan and post-scan, i.e. low, intermediate and high.

The disagreement in the assessment of pre-scan and post-scan probability $\mathrm{PE}$ was classified according to the degree of difference between nuclear medicine and referring physicians. 0 : no difference; minor: 1 grade difference interpreted as follows: normal vs. low or (vice versa), low vs. intermediate or (vice versa), intermediate vs. high (or vice versa); major: 2 grades difference interpreted as follows: low vs. high or (vice versa), intermediate vs. normal, high vs. normal or (vice versa).

The assessment of pre- and post-scan probability agreement between the nuclear medicine physicians and the referring physicians was done using the Kappa statistics. Agreement was considered poor with $\kappa \leq 0.20$ while $\kappa \geq 0.41-0.80$ indicated moderate to good agreement.

\section{Results}

The V/Q scan interpretation was normal in $12(15 \%)$ studies, low probability in 47 (59\%), intermediate probability in 7 (9\%) and high probability in $13(16 \%)$.

The nuclear medicine and referring physicians' agreement on the clinical probability of PE was moderate (63\%) before V/Q scan test which turned into good agreement (90\%) after the V/Q scan probability was known to them (table 1). Most of the nuclear medicine and referring 
Table 2. Pre-scan likelihood agreement to scan probability

\begin{tabular}{cllll}
\hline Physicians & Agreement Kappa & $\begin{array}{l}\text { Minor } \\
\text { value }\end{array}$ & $\begin{array}{l}\text { Major } \\
\text { disagreement }\end{array}$ \\
\hline disagreement
\end{tabular}

Figures in parentheses in the kappa value column are standard errors.

physicians' disagreements on PE probability, both before and after V/Q scan testing, were only of a minor degree.

The pre-scan PE likelihood assessment by the nuclear medicine physician was more in agreement with the V/Q scan PE probability than that of the referring physician (table 2). On the other hand, the post-scan PE likelihood assessments by both the nuclear medicine and the referring physician were more in agreement with the PE probability determination by the V/Q scan compared with the pre-scan probability, probably reflecting the effect of scan probability on the final impression (table 3).

\section{Discussion}

In reporting V/Q scans, the distinction between scan and post-scan probabilities is crucial for proper communication with the referring physician. Accordingly, the scan should be reported without incorporating the clinical data. Kemp et al. [7] cautioned against using clinical information in the interpretation of V/Q scans. Since the interpretation of the scan report is frequently misinterpreted by referring physicians, it may be an additional help in many situations to report post-scan probability by the nuclear medicine physician.

There is no agreement however, whether nuclear medicine physicians can report post-scan probability, that could help further in patient management. In the previous investigations of PE studies [2, 4, 5, 7, 12, 13], this question was not addressed. However, our study is addressing the above question by comparing the assessment of pre- and post-scan clinical probabilities by both nuclear medicine and referring physicians. The study shows excellent agreement of $90 \%$ between nuclear and referring physicians in assessing the post-scan probability. The disagreement in the pre-scan clinical odds was higher at 37\%
Table 3. Post-scan likelihood agreement to scan probability

\begin{tabular}{lllll}
\hline Physicians & Agreement & $\begin{array}{l}\text { Kappa } \\
\text { value }\end{array}$ & $\begin{array}{l}\text { Minor } \\
\text { disagreement }\end{array}$ & $\begin{array}{c}\text { Major } \\
\text { disagreement }\end{array}$ \\
\hline $\begin{array}{c}\text { Nuclear } \\
\text { medicine }\end{array}$ & $70 / 79$ & 0.741 & $8 / 79$ & $1 / 79$ \\
Referring & $64 / 79$ & $(0.079)$ & $(10 \%)$ & $(1 \%)$ \\
& $(81 \%)$ & $(0.557$ & $13 / 79$ & $2 / 79$ \\
& & $(16 \%)$ & $(3 \%)$ \\
\hline
\end{tabular}

Figures in parentheses in the kappa value column are standard errors.

(table 1). However, this disagreement was minor: only 1 , 5 and $10 \%$ in pre- and post-scan probabilities, respectively.

A limitation of our study is the lack of correlation with angiographic data which, if available, could have helped in clarifying further the clinical significance of the findings of the study. However, the objective of this study was to compare the clinical impressions of nuclear and referring physicians on the same cases. Therefore, angiographic or final diagnosis was not necessary to achieve the objective of the study.

\section{Conclusion}

Our data indicate that nuclear medicine physicians are capable of determining the post-scan probability of $\mathrm{PE}$ using scan and pre-scan probabilities. The scan and postscan probabilities should, however, be reported with a clear distinction to the referring physicians in order to avoid confusion in the appreciation of the final result of this test. 


\section{References}

1 Biello DR: Radiologic (scintigraphic) evaluation of patients with suspected pulmonary thrombo-embolism. JAMA 1987;257:32573259.

-2 PIOPED investigators: Value of the ventilation/perfusion scan in acute pulmonary embolism: Results of the prospective investigation of pulmonary embolism diagnosis (PIOPED). JAMA 1990;263:2753-2759.

$>3$ The PISA-PED investigators: Invasive and noninvasive diagnosis of pulmonary embolism, preliminary results of the prospective study of acute pulmonary embolism diagnosis. Chest 1995;107:335-385.

4 Gotschalk A, Sostman HD, Coleman RE, et al: Ventilation perfusion scintigraphy in the PIOPED study. II. Evaluation of the scintigraphic criteria and interpretation. J Nucl Med 1993;34:1119-1126.
5 Stein PD, Gottschalk A, Henry JW, Shivkumar K: Stratification of patients according to prior cardiopulmonary disease and probability assessment based on number of mismatched segmental equivalent perfusion defects. Approaches to strengthen the diagnostic value of ventilation/perfusion lung scans in acute pulmonary embolism. Chest 1993;104:14611467.

6 Elgazzar AH: Scintigraphic diagnosis of pulmonary embolism: Unraveling the confusion seven years after PIOPED; in Freeman LM (ed): Nuclear Medicine Annual 1997. Lippincott, Williams \& Wilkins, 1997, pp 69-101.

7 Kemp PM, Tarver DS, Batty V, Lewington V: Pulmonary Embolism. Is the clinical history a useful adjunct to aid the interpretation of equivocal lung scan? Clin Nucl Med 1996;21: 203-207.

8 McNeil BJ: Ventilation perfusion studies and the diagnosis of pulmonary embolism: Concise communication. J Nucl Med 1980;21:318323. $\checkmark 9$ Saenger EL, Bucher CR, Speaker BL, McDevitt RA: Determination of clinical efficiency: Nuclear Medicine as applied to lung scanning. J Nucl Med 1985;26:793-806.

10 Strashun A, Kean-Chuda M, Mitty H, Goldsmith SJ: V/Q scintigraphy and pre-test clinical factors to diagnose pulmonary embolism. J Nucl Med 1986;27:922-923.

11 Kember PG, Euiton HA, Morcos SK: Clinician's interpretation of the indeterminate ventilation-perfusion scans report. Br J Radiol 1997; 70:1109-1111.

12 Worsley DF, Alavi A: Comprehensive analysis of the results of the PIOPED study. J Nucl Med 1995;36:2381-2387.

13 Worsley DF, Kim CK, Alavi A, Polansly HI: Detailed analysis of patients with matched ventilation perfusion defects and chest radiographic opacities. J Nucl Med 1993;34:18511853. 